\title{
Hypodysfibrinogenemia in a young patient with recurrent strokes
}

\author{
Daniele Orsucci ${ }^{1}$, Stefania Salvetti ${ }^{1}$, Marco Margelli², Michele Puglioli ${ }^{3}$, Marco Vista ${ }^{1}$, Monica Mazzoni ${ }^{1}$ \\ 'Unit of Neurology, San Luca Hospital, Lucca 55100, Italy. \\ ${ }^{2}$ Unit of Transfusional Medici-ne and Immunohematology, San Luca Hospital, Lucca 55100, Italy. \\ ${ }^{3}$ Unit of Neuroradiology, University Hospital of Pisa, Pisa 56100, Italy. \\ Correspondence to: Dr. Daniele Orsucci, Unit of Neurology, San Luca Hospital, Via Lippi-Francesconi, Lucca 55100, Italy. \\ E-mail: orsuccid@gmail.com
}

How to cite this article: Orsucci D, Salvetti S, Margelli M, Puglioli M, Vista M, Mazzoni M. Hypodysfibrinogenemia in a young patient with recurrent strokes. Neuroimmunol Neuroinflammation 2018;5:6. http://dx.doi.org/10.20517/2347-8659.2017.66

Received: 23 Dec 2017 Frist Decision: 6 Feb 2018 Revised: 11 Feb 2018 Accepted: 12 Feb 2018 Published: 7 Mar 2018

Science Editor: Athanassios P. Kyritsis Copy Editor: Jun-Yao Li Production Editor: Huan-Liang Wu

\begin{abstract}
Stroke is a complex disease, but in some instances is the direct result of a monogenic disease. Here we report the case of a 44-year-old Italian man who experienced recurrent transitory ischemic attacks and strokes. He also had right fetaltype posterior cerebral artery. He was diagnosed with congenital hypodysfibrinogenemia due to a mutation leading to a truncated fibrinogen gamma chain. Further studies are needed to better elucidate the links between fibrinogen dysfunction and stroke. Hypodysfibrinogenemia should be included among the monogenic diseases associated with ischemic stroke. Physicians should be aware of this condition, which may be detectable on routine assays.
\end{abstract}

Keywords: Coagulation, dysfibrinogenemia, fibrinogen, genetics, ischemic stroke, monogenic

\section{INTRODUCTION}

Stroke is a complex disease resulting from the interplay of genetics and environment. In some instances stroke is the direct result of a monogenic disease, mainly in young adults. Here we report the case of a 44-year-old Italian man who experienced recurrent transitory ischemic attacks (TIAs) and strokes from the age of 38 years. At last, he was diagnosed with congenital hypodysfibrinogenemia due to a mutation leading to a severely truncated fibrinogen gamma chain.

\section{CASE REPORT}

Our patient presented at 38 years with recurrent TIAs ( 1/month), characterized by sensory disturbances

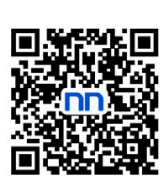


on the left side of the body. Both the family history and past medical history were unremarkable. At that time, brain magnetic resonance imaging (MRI) was normal except few non-specific white matter lesions. Electroencephalography (EEG) (including prolonged monitoring) was normal. He was prescribed acetylsalicylic acid (ASA) $100 \mathrm{mg} /$ day, but chose to discontinue using after an episode of nosebleeding.

At age 40 years, he presented with left hemiparesis due to a right mesial frontal ischemic stroke [Figure $1 \mathrm{~A}$ and B]. The patient, who was prescribed with ASA $300 \mathrm{mg} /$ day, gradually recovered until the following year (aged 41 years), when he was examined because of acute-onset left hemianopia. MRI showed an acute ischemic lesion in the right temporo-parietal-occipital regions [Figure $1 \mathrm{C}$ and $\mathrm{D}$ ]. Ivy bleeding time was prolonged to $14 \mathrm{~min}$ (normal 2.5-11 $\mathrm{min}^{[1]}$ ) confirming appropriate antiaggregation.

Cerebrovascular angiography, performed under the hypothesis of a vasculitis, showed only a right fetaltype posterior cerebral artery (PCA) [Figure $1 \mathrm{E}$ and F]. Brain 18F-fluorodeoxyglucose positron emission tomography and cerebrospinal fluid examination were unremarkable. Complete cardiologic screening was normal. EEG showed mild epileptiform alterations on the right hemisphere. Cerebral autosomal dominant arteriopathy with subcortical infarcts and leukoencephalopathy (CADASIL), Fabry disease and mitochondrial disorders were excluded by appropriate biochemical and genetic studies.

Routine laboratory assays (including D-dimer, prothrombin time, activated partial thromboplastin time, factor $\mathrm{V}$, antithrombin, protein $\mathrm{C}$, protein $\mathrm{S}$, activated protein $\mathrm{C}$ resistance, lupus anticoagulans, antiantinuclear antibodies, anti-smooth muscle antibodies, anti-cardiolipin antibodies, extractable nuclear antigen antibodies, anti-neutrophil cytoplasmic antibodies and antiphospholipid antibodies) were normal, except for a mild but long-lasting hypofibrinogenemia (115-180 mg/dL; normal 200-400 mg/dL).

Hypofibrinogenemia is most often caused by heterozygous mutations in one of the three fibrinogen chains, whereas the same homozygous mutations commonly lead to afibrinogenemia, a bleeding disease. Most cases of heterozygous disorders of fibrinogen affect quantity and quality of circulating fibrinogen (hypodysfibrinogenemia, OMIM \#616004), and may lead to both bleeding diathesis and thromboembolic complications (even in the same patient). This predisposition for thrombosis has been ascribed to delayed plasmin digestion $^{[2]}$.

Ischemic stroke was reported only in exceptional patients with homozygous gamma chain (FGG) mutations ${ }^{[3]}$, but large case-control association studies showed that polymorphisms in the genes encoding the alpha and gamma chains may lead to an increased risk of ischemic stroke, likely mediated by qualitative effects on fibrinogen (and fibrin) ${ }^{[4]}$.

These considerations prompted a genetic work-up in our patients. At last, sequencing of the three fibrinogen chains showed the $945 \mathrm{C}>\mathrm{T}$ mutation in the FGG gene, leading to a stop codon (p.Arg108Stop of the mature chain). This mutation was previously reported in ten consanguineous, homozygous afibrinogenemic patients from Lebanon ${ }^{[5]}$. These patients had bleeding diathesis. Unfortunately, no detailed clinical data on the heterozygous relatives were provided. However they had, as our patients, mild hypofibrinogenemia with other coagulation tests in the normal range. Of note, expression studies demonstrated that this nonsense mutation affected neither mRNA splicing nor stability, but led to the production of an unstable, severely truncated fibrinogen gamma chain that is not incorporated into a functional fibrinogen hexamer (in this study the mutation was denoted as p.Arg134Stop, considering the immature chain $)^{[5]}$.

The genetic finding confirmed the diagnosis of congenital hypodysfibrinogenemia in our patient, who in the 3 years following the 2nd stroke did not experience further TIAs or stroke (under double-antiaggregation therapy with ASA $100 \mathrm{mg} /$ day and clopidogrel $75 \mathrm{mg} /$ day). He had only 3 episodes of simple motor partial seizures successfully treated with oxcarbazepine. 

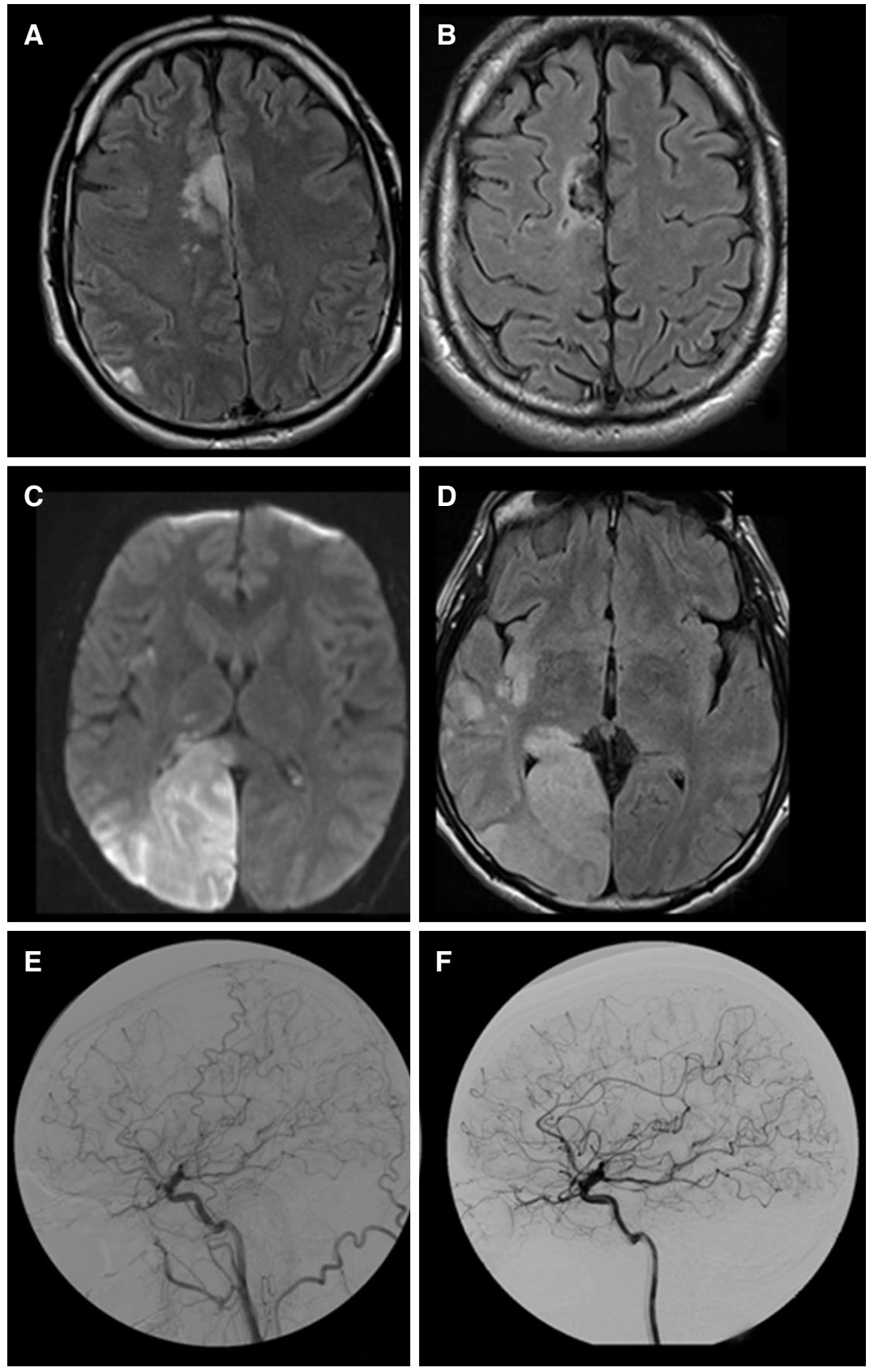

Figure 1. Recurrent strokes and fetal-type PCA in our patient. (A, B) Right mesial frontal ischemic stroke at age 40 years, and its 6-month evolution (FLAIR axial MRI images); (C, D) acute ischemic lesion in the right temporo-parietal-occipital regions at age 41 years (diffusionweighted and FLAIR axial MRI images); (E, F) two consecutive angiograms (at the time of the first stroke and after 6 months) showing the fetal-type right PCA, originating from the right internal carotid artery. PCA: posterior cerebral artery; MRI: magnetic resonance imaging

\section{DISCUSSION}

To our knowledge, this is the first report of an heterozygous fibrinogen mutation in a young patient with recurrent TIAs and strokes. Further studies are needed to better elucidate the links between fibrinogen function (and dysfunction) and ischemic stroke. It is likely other genetic and/or environmental factors may have a role.

From this perspective, an intriguing observation is the lateralization of all the TIAs and strokes of our patient in the right hemisphere (in the presence of a right fetal-type PCA). Even if the association between 
fetal PCA and ischemic stroke is debated ${ }^{[6]}$, fetal PCA may have some hemodynamic impact (e.g. faster perfusion transit times ipsilateral to fetal origin of the PCA ${ }^{[7]}$, and a detrimental interaction of the two factors (hemodynamics and coagulation) cannot be ruled out.

In conclusion, hematologists, geneticists and neurologists should be aware of this condition, which in most cases may easily be detectable on routine assays, and hypodysfibrinogenemia should be included among the monogenic diseases ${ }^{[8]}$ associated with ischemic stroke in younger adults.

\section{DECLARATIONS}

\section{Authors' contributions}

Prepared the manuscript: Orsucci D

Revised the manuscript: Mazzoni M

Clinically studied the patient: Salvetti S, Vista M

Performed the hematological examinations: Margelli M

Performed the angiographic studies: Puglioli $M$

\section{Financial support and sponsorship}

None.

\section{Conflicts of interest}

There are no conflicts of interest.

\section{Patient consent}

The manuscript does not contain patient identifiable data.

\section{Ethics approval}

Not applicable.

\section{Copyright}

(c) The Author(s) 2018.

\section{REFERENCES}

1. Mielke CH Jr, Kaneshiro MM, Maher IA, Weiner JM, Rapaport SI. The standardized normal Ivy bleeding time and its prolongation by aspirin. Blood 1969;34:204-15.

2. Asselta R, Duga S, Tenchini ML. The molecular basis of quantitative fibrinogen disorders. J Thromb Haemost 2006;4:2115-29.

3. Lounes KC, Soria C, Mirshahi SS, Desvignes P, Mirshahi M, Bertrand O, Bonnet P, Koopman J, Soria J. Fibrinogen Alès: a homozygous case of dysfibrinogenemia (gamma-Asp(330)-->Val) characterized by a defective fibrin polymerization site "a". Blood 2000;96:3473-9.

4. Jood K, Danielson J, Ladenvall C, Blomstrand C, Jern C. Fibrinogen gene variation and ischemic stroke. J Thromb Haemost 2008;6:897904.

5. Neerman-Arbez M, Germanos-Haddad M, Tzanidakis K, Vu D, Deutsch S, David A, Morris MA, de Moerloose P. Expression and analysis of a split premature termination codon in FGG responsible for congenital afibrinogenemia: escape from RNA surveillance mechanisms in transfected cells. Blood 2004;104:3618-23.

6. Arjal RK, Zhu T, Zhou Y. The study of fetal-type posterior cerebral circulation on multislice CT angiography and its influence on cerebral ischemic strokes. Clin Imaging 2014;38:221-5.

7. Wentland AL, Rowley HA, Vigen KK, Field AS. Fetal origin of the posterior cerebral artery produces left-right asymmetry on perfusion imaging. AJNR Am J Neuroradiol 2010;31:448-53.

8. Mancuso M, Orsucci D, Ienco EC, Brondi M, Simoncini C, Chiti A, Montano V, Terni E, Giannini N, Siciliano G, Bonuccelli U. Common genetic conditions of ischemic stroke to keep in mind. Curr Mol Med 2014;14:979-84. 